doi:10.17659/01.2020.0041

Journal of Case Reports 2020;10(3):154-157

\title{
Non-Hodgkin's Lymphoma of Spleen: A Rare Site of Primary Presentation
}

\author{
Manishi Bansal ${ }^{1}$, Smriti Gupta $^{2}$, Girdhar Gopal ${ }^{3}$, Ankush Jindal ${ }^{4}$ \\ Departments of ${ }^{1}$ Radiotherapy, ${ }^{2}$ Pathology and ${ }^{3}$ Oncosurgery, Mayo Hospital, Mohali; ${ }^{4}$ Government Medical College and Hospital, \\ Chandigarh, India.
}

\section{Corresponding Author: \\ Dr. Manishi Bansal \\ Email: manishi1@yahoo.com}

This is an Open Access article distributed under the terms of the Creative Commons Attribution License (creativecommons.org/ licenses/by/3.0).

Received

Accepted

Published

May 13, 2020

July 13,2020

July 30,2020

\begin{abstract}
Background: Isolated splenic involvement in non-Hodgkin's lymphoma is a rare clinical entity and is termed as primary splenic lymphoma (PSL). In such cases, the disease is mostly confined to spleen and splenic hilar lymph nodes. It usually presents with dragging pain in left side of abdomen and huge splenomegaly is detected on investigations. Splenectomy is the main modality of treatment followed by chemotherapy with or without radiation. Case Report: We present a rare case of PSL treated successfully at our institution with splenectomy and chemotherapy. Conclusion: Primary splenic lymphoma is a rare splenic neoplasm. Immunohistochemistry markers are helpful in confirming the diagnosis. Splenectomy is both diagnostic and therapeutic in such cases.
\end{abstract}

Keywords: Primary Splenic Lymphoma, Non-Hodgkin's Lymphoma, Splenectomy, Splenic radiation, Diffuse Large B-cell Lymphoma.

\section{Introduction}

Spleen is an organ which is usually involved in malignancies which are diffuse and disseminated. Spleen is frequently involved as a secondary lymphoid organ. Neoplasm originating from the spleen per se is a rare occurrence, especially as part of lymphomas which are characterized by widespread organ and lymph nodes involvement [1]. By definition, primary splenic lymphomas (PSL) are lymphomas involving spleen only with or without splenic hilar lymph nodes. PSL comprises of only $1 \%$ of all non-Hodgkin lymphoma (NHL) and are predominantly of B-cell origin [2]. Secondary involvement of spleen as part of lymphoma and other malignancies is a common phenomenon. We hereby report a rare case of primary B-cell NHL of spleen.

\section{Case Report}

A 60 year old female presented with diffuse pain in the left side of abdomen, fatigue and weight loss. Her examination revealed a large palpable spleen in absence of hepatomegaly or any other peripheral lymph nodes. Routine blood counts showed microcytic anaemia whereas liver, kidney function test and chest X-ray were normal. Ultrasound abdomen revealed huge splenomegaly measuring $13.9 \mathrm{~cm}$ with a heterogenous hypoechoic mass measuring $7.9 \times 9.7 \mathrm{~cm}$ in splenic parenchyma. CT scan revealed a large relatively well marginated heterogeneously hypodense mass in the upper and mid-polar regions of the spleen. Positron Emission Tomography-Computed Tomography (PET-CT) revealed intense fluorodeoxyglucose (FDG) uptake of SUVmax 40.6, heterogeneously enhancing partially exophytic, lesion arising from upper-mid polar region of spleen measuring $9.8 \times 9.8 \times 11.5 \mathrm{~cm}$ with internal necrosis and FDG uptake in few perisplenic lymph nodes, largest measuring $17 \mathrm{~mm}$. Her bone marrow examination showed mildly hypercellular marrow with normal hematopoietic elements. Viral markers were negative. Case was discussed in tumor board and the decision for splenectomy was taken. 
Gross examination of the specimen showed a greyish-white mass in the upper and mid-pole of spleen with areas of necrosis within. The splenic capsule was intact. Histopathology revealed splenic tissue with sheets of predominantly medium to few scattered large sized lymphoid cells, with brisk mitosis and foci of necrosis [Fig.1]. Immunohistochemistry revealed that the tumor was positive for LCA, CD20, Pax5 and BCL2 and negative for CD3, CD 15, BCL6, CD5 [Fig.2,3]. The ki-67 index was 20-25\% [Fig.4]. A diffuse large B cell lymphoma (DLBCL) was diagnosed. She was treated with 6 cycles of R-CHOP chemotherapy which she tolerated well. Presently, she is asymptomatic and disease free on PET CT scans after one year of follow up.

\section{Discussion}

Primary splenic tumors are a rare entity and include malignant lymphomas, angiosarcomas and lymphangiomas. Primary splenic lymphoma has been described both in context of Hodgkin's and non-Hodgkin's lymphoma [3]. Non-Hodgkin's lymphoma (NHL) is usually a clinical entity with diffuse involvement of lymph nodes, liver and spleen. Isolated involvement of any organ is a rare occurrence especially of spleen accounting for only $1 \%$ of all NHLs. In one series, out of 500 splenectomy specimens with malignant lymphoma, only 17 patients $(3.4 \%)$ were found to be consistent with the diagnosis of primary splenic lymphoma [4]. Primary splenic lymphoma is defined differently by different authors. Dasgupta defined PSL as NHL confined only to spleen and splenic hilar lymph nodes [5], as in our case, Kehoe and Straus defined PSL as disease involving spleen, splenic hilar lymph nodes and other lymph nodes as well [6]. However, Skarin et al. suggested that diagnosis of PSL can be made if splenomegaly is the predominant feature in any lymphoma [7].

The usual presenting symptoms of PSL are diffuse and dragging pain in the left side of abdomen, fatigue, weight loss, fever and night

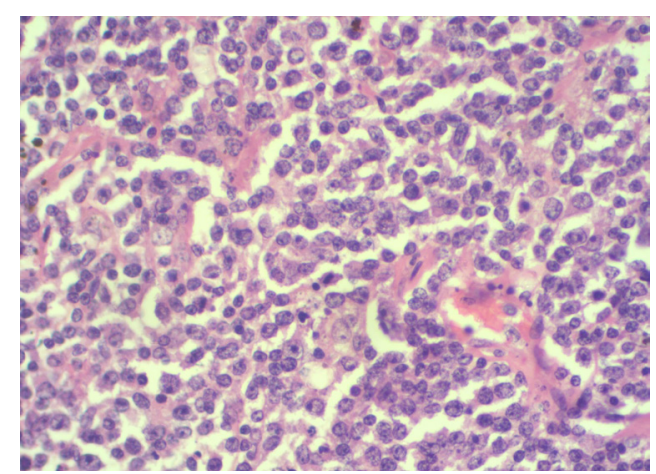

Fig.1: $H \& E$ 40X magnification showing medium to large sized lymphoid cells.

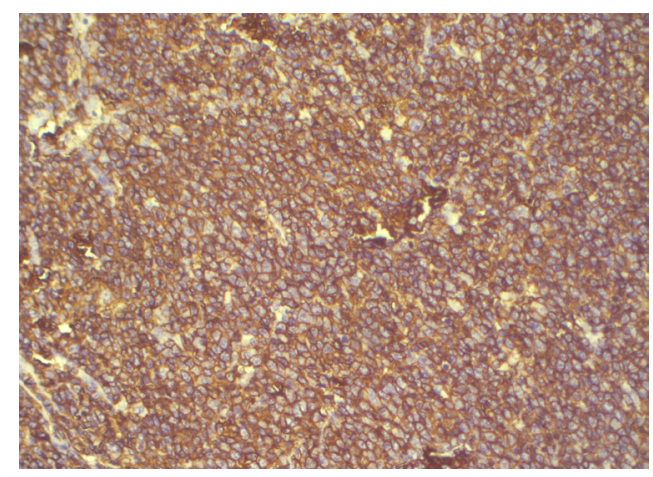

Fig.2: CD 20 showing membranous positivity.

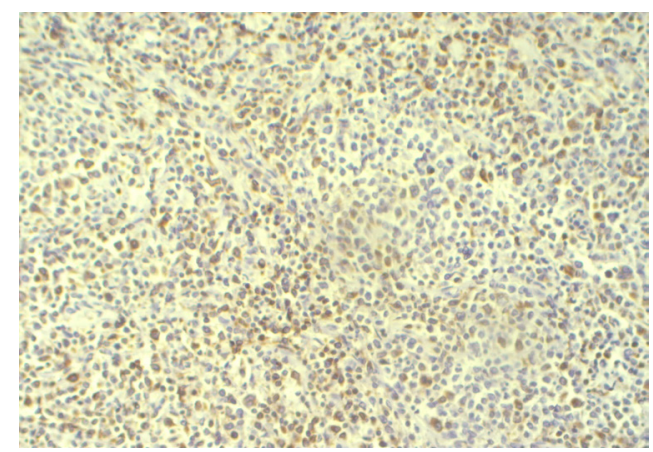

Fig.3: $B C L 2$ is patchy positive.

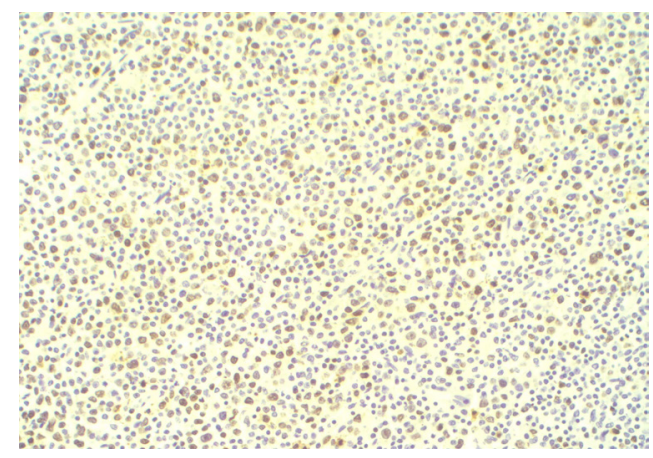

Fig.4: Ki 67 index of 20-25\%. 
sweats. Our patient also presented with pain in left side of abdomen and fatigue. Blood picture might reveal cytopenia in advanced cases as in our case microcytic anemia was seen. Ultrasound and CT scan of abdomen are most valuable in diagnosing PSL. PET-CT scan is useful in differentiating PSL from a generalized form of NHL. The most common histological subtypes are low or intermediate grade lymphocytic lymphoma, mantle cell lymphoma and diffuse large B-cell lymphoma which is seen in $30 \%$ of cases [8]. Our patient also has diffuse large B-cell lymphoma (DLBCL) type of NHL. DLBCL of spleen are aggressive neoplasm and usually present with single or multiple confluent nodules derived from white pulp [9]. They commonly present in sixth and seventh decade without bone marrow involvement. The diagnosis is usually made by imaging methods, fine needle aspiration or core biopsy from spleen. Immunohistochemical studies are done for confirmation and sub-typing of NHL.

Histomorphologically, DLBCL is a neoplasm of medium to large B lymphoid cells whose nuclei are the same size or larger than those of normal macrophages or more than twice the size of those of normal lymphocytes with a diffuse growth pattern [10]. It is also suggested that hepatitis C virus may have a role in lymphomagenesis of splenic DLBCL [1]. The morphology of DLBCL is diverse and disease can be divided into common and rare morphological variant, hence ancillary studies are critical before making diagnosis. Denovo CD5 positivity is variably reported to behave prognostic importance. It is often associated with high risk clinical features, especially in Asian countries [10]. In our case CD5 was negative. The prognostic value of $\mathrm{Ki}-67$ in DLBCL has been analyzed in various studies and it has been seen that a high Ki-67 index predicted worse survival, especially in the Rituximab era [11].

A staging system of PSL was also proposed by Ahmann et al. depending on the extent of disease [12]. Stage I refers to patients with disease limited to spleen only, stage II is involvement of spleen and splenic hilar lymph nodes, while stage III is disease spread beyond splenic hilum. Our patient belonged to stage II. The prognostic factors of PSL are stage of disease at presentation, histological type of tumor, performance status and Ki-67 expression [13]. DLBCL is one of the aggressive types.

The main modality of treatment of PSL is splenectomy, which is both diagnostic as well as therapeutic as in our case. Splenectomy helps in reducing the disease burden instantly, helps in subjective improvement and also improves survival rates. In a retrospective study of 87 patients by Bairey et al. overall survival at 5 years was $91 \%$ for splenectomized patients as compared to $68 \%$ for non-splenectomized patients [11]. Although this difference was not statistically significant but was more apparent for early stage disease. Chemotherapy is usually preferred after splenectomy to decrease chances of relapse. R-CHOP or CHOP is the standard protocol followed like in other NHL after cardiac workup [14]. It is well tolerated with minimal side effects. Patient who is unfit for splenectomy or if primarily unresectable, can be down staged with chemotherapy and later considered for surgery if possible. The role of tumor bed radiation is limited in splenectomised patients and is used only for patients who have bulky residual disease or who are unfit for chemotherapy. For non-splenectomised patients, splenic radiation may be used with or without chemotherapy to reduce the size of the tumor [11].

\section{Conclusion}

Primary splenic lymphoma constitutes a rare entity of splenic neoplasms and IHC markers are helpful in confirming the exact diagnosis. Splenectomy is both diagnostic and therapeutic in such cases. Adjuvant chemotherapy or radiotherapy is mostly needed. Prognosis depends on stage and histology of the tumor. 
Contributors: MB: manuscript editing, patient management and the conception of the work; SG: manuscript writing, literature review and histopathology; GG: patient management and critical review; AJ: literature review and references. MB will act as a study guarantor. All authors approved the final version of this manuscript and are responsible for all aspects of this study.

Funding: None; Competing interests: None stated.

\section{References}

1. Jerome S. Burke. Spleen. In: Stacey E. Mills (ed). Sternberg's Diagnostic Surgical Pathology $6^{\text {th }}$ edition. Philadelphia: Wolters Kluwer. 2015; p.1216-1224.

2. Wu CM, Cheng LC, Lo GH, Lai KH, Cheng CL, Pan WC. Malignant lymphoma of spleen presenting as acute pancreatitis. World J Gastroenterol. 2007;13:3773-3775.

3. Konstantiadou I, Mastoraki A, Papanikolaou I, Sakorafas G, Safioleas M. Surgical approach of primary splenic lymphoma: Report of a case and review of literature. Indian J Hematol Blood Transfusion. 2009;25:120-124.

4. Falk S, Stutte HJ. Primary malignant lymphoma of the spleen. A morphologic and immunohistochemical analysis of 17 cases. Cancer. 1990;66:2612-2619.

5. Dasgupta T, Coombes BC, Brasfield RD. Primary malignant neoplasms of the spleen. Surg Gynaecol Obstet. 1965;120:947-960.
6. Kehoe J, Straus DJ. Primary lymphoma of the spleen. Clinical features and outcome after splenectomy. Cancer. 1988;62:1433-1438.

7. Skarin AT, Davey FR, Moloney WC. Lymphosarcoma of spleen. Arch Intern Med. 1971;127:259-265.

8. Gobbi P, Grignani G, Pozzetti U, Bertolini D, Pieresca C, Montagna G, et al. Primary splenic lymphoma: Does it exist? Haematological. 1994;79:286-293.

9. Kashmura M, Naro M, Akikusa B, Okuhara A, Momose $\mathrm{H}$, Miura I, et al. Primary splenic DLBCL manifesting in red pulp. Virchows Arch. 2008;453:501-509.

10. Pris MA, Campo E. Splenic B-cell lymphoma/leukemia. In: Swedlow SH (ed). WHO classification of tumours of haematopoetic and lymphoid tissues. Revised $4^{\text {th }}$ edition. Lyon, 2017. pp.229.

11. Bairey O, Shvidel L, Perry C, Dann EJ, Ruchlemer $\mathrm{R}$, Tadmor $\mathrm{T}$, et al. Characteristics of primary splenic diffuse large B-cell lymphoma and role of splenectomy in improving survival. Cancer. 2015;1:2909-2916.

12. Ahmann DL, Kiely JM, Harrison EG, Payne WS. Malignant lymphoma of spleen. Cancer. 1966;19:461469.

13. Kattepur AK, Rohith S, Shivaswamy S Babu R, Santhosh. Primary splenic lymphoma: A case report. Indian J Surg Oncol. 2013;4:287-290.

14. Iannitto E, Tripodo C. How I diagnose and treat splenic lymphomas. Blood. 2011;117: 2585-2595. 